\title{
Argumentos de contraste fenoménico a favor de la fenomenología cognitiva*
}

\section{Phenomenal contrast arguments for cognitive phenomenology}

\author{
Phenomenal contrast arguments \\ Trad.: Juan Fernando Álvarez Céspedes \\ Instituto de Filosofía \\ Universidad de Antioquia \\ Medellín, Antioquia \\ E-mail: juan.alvarez29@udea.edu.co
}

\author{
Por: Elijah Chudnoff \\ Department of Philosophy \\ University of Miami \\ Miami, Estados Unidos \\ E-mail: e.chudnoff@miami.edu \\ Trad.: Elizabeth Cardona Muñoz \\ Instituto de Filosofía \\ Universidad de Antioquia \\ Medellín, Antioquia
}

E-mail: elizabeth.cardonam@udea.edu.co

Fecha de recepción: $10^{\circ}$ de noviembre de 2017

Fecha de aprobación: 30 de noviembre de 2017

Doi: 10.17533/udea.ef.n57a09

Resumen. De acuerdo con quienes proponen que la fenomenología cognitiva es irreductible, algunos estados cognitivos ocasionan estados fenoménicos en el sujeto para los que no son condición suficiente los estados totalmente sensoriales. Uno de los enfoques principales para defender el punto de vista de que hay fenomenología cognitiva irreductible consiste en ofrecer un argumento de contraste fenoménico. En este artículo distingo tres tipos de argumentos de contraste fenoménico: el que llamo puro — representado por el argumento Jack/Jacques de Strawson-, el hipotético — representado por el argumento de Zoe de Kriegel - y el glosado —desarrollado por primera vez aquí. Afirmo que los argumentos de contraste fenoménico puro e hipotético enfrentan dificultades significativas, pero hay un argumento sólido de contraste glosado a favor de la fenomenología cognitiva irreductible.

Palabras clave: Cognición, fenomenología, contraste fenoménico, Strawson, Kriegel

Abstract. According to proponents of irreducible cognitive phenomenology some cognitive states put one in phenomenal states for which no wholly sensory states suffice. One of the main approaches to defending the view that there is irreducible cognitive phenomenology is to give a phenomenal contrast argument. In this paper I distinguish three kinds of phenomenal contrast argument: what I call pure - represented by Strawson's Jack/Jacques argument — hypothetical_- represented by Kriegel's Zoe argument — and glossed - first developed here. I argue that pure and hypothetical phenomenal contrast arguments face significant difficulties, but that there is a sound glossed phenomenal contrast argument for irreducible cognitive phenomenology.

Keywords: Cognition, phenomenology, phenomenal contrast, Strawson, Kriegel

* Esta traducción fue elaborada con la ayuda del autor Elijah Chudnoff. Agradecemos a la revista Philosophy and Phenomenological Research por dar los permisos necesarios para elaborar la traducción de este artículo (N. T.)

Como citar este artículo:

MLA: Chudnoff, Elijah. "Argumentos de contraste fenoménico a favor de la fenomenología cognitiva”. Trad.: Chudnoff, Elijah, Cardona, Elizabeth, Álvarez, Juan Fernando. Estudios de Filosofía, 57 (2018): 175-203

APA: Chudnoff, E. (2018). Argumentos de contraste fenoménico a favor de la fenomenología cognitiva. Trad.: Chudnoff, E.; Cardona, E.; Álvarez, J. F. Estudios de Filosofía. (57), 175-203.

Chicago: Chudnoff, E. "Argumentos de contraste fenoménico a favor de la fenomenología cognitiva." Trad.: Chudnoff, E.; Cardona, E.; Álvarez, J. F. Estudios de Filosofía n. ${ }^{\circ} 57$ (2018): 175-203. 
Imagine estar en las siguientes situaciones:

[Comprender] Usted está tratando de leer las instrucciones de una medicina que un veterinario ha prescrito para su perro. Al principio son ilegibles. Luego usted ve que dice que se debe administrar la medicina dos veces al día durante una semana.

[Intuir] Usted lee en un libro, "Si a $<1$, entonces $2-2 \mathrm{a}>0$ ", y se pregunta si esto es verdadero. Luego, usted "ve" cómo el hecho de que "a" sea menor que 1 hace que 2a sea menor que $2 \mathrm{y}$, por tanto, que $2-2 \mathrm{a}$ sea mayor que 0 .

[Ver] Usted está buscando sus perros en el parque. Al principio, no puede distinguirlos de la multitud de los otros perros. Pero luego los ve persiguiendo una pelota de tenis.

[Reaccionar] En las noticias usted lee acerca del colapso de una fábrica sobre trabajadores textiles de Bangladesh, a quienes se les ordenó trabajar a pesar de las advertencias sobre la seguridad del edificio. Esto lo hace sentir triste y enfadado.

En las primeras dos de estas situaciones hay un cambio en el estado cognitivo. Al principio usted no comprende o intuye, y después lo hace. En las otras dos situaciones hay un cambio en el estado sensorial general. Al principio usted no ve o no reacciona emocionalmente, y luego usted lo hace.

En todas estas situaciones hay un cambio en el estado fenoménico. Para usted, hay algo que "es como" antes de comprender, intuir, ver o reaccionar. Hay algo que es como al momento de comprender, intuir, ver o reaccionar. $Y$ cómo es antes de estar en estos estados mentales es diferente de cómo es mientras se está en ellos.

La pregunta principal en la literatura reciente sobre fenomenología cognitiva, en una primera aproximación, es esta: ¿son los cambios en los estados fenoménicos exhibidos en los primeros dos casos de un tipo diferente de los cambios en estados fenoménicos exhibidos en los otros dos casos? Los proponentes de la fenomenología cognitiva irreductible dicen que sí. Los oponentes dicen que no.

Quienes proponen la fenomenología cognitiva irreductible han apelado a una cantidad de argumentos diferentes a favor de su punto de vista. Algunos son argumentos que apelan a la mejor explicación del acceso introspectivo que tiene el sujeto a sus estados cognitivos, otros son argumentos del interés o del valor de los estados cognitivos y otros son argumentos del rol de los estados cognitivos en la fundamentación de la intencionalidad. ${ }^{2}$ Mi propósito en este artículo es explorar las

1 Esta expresión, que no es muy clara en español, proviene del famoso texto de Thomas Nagel "What is it like to be a bat" (1974). Esta expresión a veces se traduce como "hay algo que se se siente..."; sin embargo, aquí seguimos la traducción propuesta en la edición en español de Ezcurdia y Hansberg (2003) (N. T.).

2 Véase, por ejemplo, Pitt (2014) para un argumento desde la introspección, Strawson (2011) para un 
perspectivas de un grupo diferente de argumentos que son denominados argumentos de contraste fenoménico. ${ }^{3}$

En este artículo distingo tres clases de argumento de contraste fenoménico. Todos dependen de premisas sobre los caracteres fenoménicos de algunos estados mentales. Los argumentos de la primera clase dependen solamente de premisas sobre las diferencias fenoménicas entre estados mentales. A estos los llamaré argumentos de contraste fenoménico puro. Desde mi punto de vista, estos argumentos no son fuertes. Los argumentos de la segunda y tercera clase representan diferentes maneras de fortalecer el enfoque de contraste fenoménico que aboga por la fenomenología cognitiva irreductible. Los argumentos de la segunda clase descansan sobre premisas acerca de las diferencias fenoménicas entre los estados mentales de personas hipotéticas, que carecen de toda fenomenología sensorial. A estos los llamaré argumentos de contraste fenoménico hipotético. Desde mi punto de vista, estos argumentos son problemáticos. Los argumentos de la tercera clase no descansan únicamente sobre premisas acerca de las diferencias fenoménicas entre estados mentales. Estos argumentos también dependen de premisas que proveen una glosa acerca de estas diferencias fenoménicas. A estos los llamaré argumentos de contraste fenoménico glosado. Desde mi punto de vista, hay un buen argumento de contraste fenoménico glosado a favor de la Irreductibilidad.

Este es el plan. En la primera sección, a continuación, explico cómo uso términos tales como "estado fenoménico" " "fenomenología cognitiva irreductible", y distingo algunas tesis diferentes en esta área. Cada una de las siguientes tres secciones aborda una de las tres clases de argumentos de contraste fenoménico. Una sección final resume los puntos principales de la discusión.

\section{Términos y tesis}

Recuerde el ejemplo de ver planteado más arriba:

[Ver] Usted está buscando sus perros en el parque. Al principio, no puede distinguirlos de la multitud de los otros perros. Pero luego los ve persiguiendo una pelota de tenis.

argumento desde el interés y Horgan \& Graham (2012) para un argumento desde la fundamentación de la intencionalidad.

3 Los argumentos de contraste fenoménico están esparcidos a través de la literatura. Los primeros ejemplos notables incluyen a Strawson (1994), Siewert (1998), Horgan \& Tienson (2002). Estos fueron discutidos en una serie de artículos recopilados en Bayne \& Montague (2011). El término "contraste fenoménico" deriva de Susanna Siegel. Véase: Siegel $(2007,2010)$. 
Hay al menos cuatro cosas diferentes que podríamos decir sobre este caso.

Primero, se trata de un cambio en el estado mental. No trataré de decir lo que es un estado mental en términos más básicos. Lo tomaré como un primitivo. Nada dependerá de la diferencia entre estados mentales y eventos mentales, así que no los trataré separadamente.

La segunda cosa que podríamos decir sobre [Ver] es que se trata de un cambio en el estado fenoménico. Por un estado fenoménico me refiero a un estado mental que se individua por cómo es para uno estar en él. Cómo es estar en un estado mental para uno es el carácter fenoménico de ese estado mental. Y decir que su carácter fenoménico individua un estado mental es decir que no se requiere nada más ni nada menos para estar en ese mismo estado mental que estar en un estado mental con justamente el mismo carácter fenoménico.

En adición a la noción de un estado mental que se individua por su carácter fenoménico, es útil tener una noción de un estado mental que esencialmente tiene algún carácter fenoménico $u$ otro, tal vez dentro de un cierto rango restringido, pero que no se individua por cualquier carácter fenoménico particular. Tales estados los llamaré estados mentales fenoménicamente conscientes (Cf. Siegel, 2010).

La tercera cosa que podríamos decir a propósito de [Ver] es que hay un cambio en el estado fenoménicamente consciente. En cualquier ocasión particular en la que usted ve a sus perros, ellos se ven de alguna manera específica. Pero justo de qué manera se ven diferirá en ocasiones diferentes. Por ejemplo, usted podría ver sus perros de frente, desde atrás o de lado. En cada caso hay alguna manera en la que sus perros se ven para usted. Pero la manera exacta en la que ellos se ven para usted difiere de caso en caso. Sin embargo, en cada caso usted se encuentra en el estado mental de ver a sus perros. Así que el estado de ver sus perros no es un estado fenoménico. Este es un ejemplo de un estado fenoménicamente consciente.

La cuarta cosa que podríamos decir sobre [Ver] es que se trata de un cambio en el estado sensorial, en lugar de, o al menos en adición a, un cambio en el estado cognitivo. Hay maneras diferentes de entender esta distinción, y una discusión completa sobre ello tendría que ser bastante extensa. Aquí solo intentaré explicar cómo entiendo el asunto y solo diré lo suficiente para hacer útil la distinción para los propósitos presentes. ${ }^{4}$

4 Para una discusión más completa véase Chudnoff (2015). 
Contraste los dos estados mentales siguientes: pensar que hay una carta en su buzón, ver que hay una carta en su buzón. Supongamos que los estados mentales de pensar que hay una carta en su buzón y ver que hay una carta en su buzón tienen cierto contenido representacional común —algo común recogido en la cláusula "que hay una carta en su buzón". De todos modos, hay una diferencia clara entre los estados mentales: cuando usted ve que hay una carta en su buzón, esta parece estar allí frente a usted. Usted se representa que hay una carta en su buzón y al mismo tiempo es consciente de partes de su entorno inmediato que lo corroboran. El contenido del estado sensorial está ligado a lo que parece presente aquí y ahora. Esto no necesita ser así cuando usted piensa que hay una carta en su buzón. Usted podría pensar que hay una carta en su buzón aun cuando se encuentre a kilómetros de este. El contenido del estado cognitivo no depende de lo que a usted le parece presente aquí y ahora. En mi opinión, esta observación es clave para trazar la distinción entre los estados sensoriales y los cognitivos.

Transformar esto en una caracterización clara y adecuadamente general de la distinción entre estados sensoriales y cognitivos toma bastante trabajo. Sin embargo, no tengo espacio para perseguir ese proyecto aquí. Para los propósitos presentes, el siguiente esbozo debería bastar. Cuando usted piensa que hay una carta en su buzón, se representa esto de una manera que es independiente de la conciencia presente de lo que podríamos llamar un testimonio de esa proposición. Esta caracterización ofrece cierta estructura a la idea del sentido común de que el pensamiento es independiente de lo que parece presente aquí y ahora. Y más generalmente podríamos decir: un estado mental representa que $p$ de una manera cognitiva solo en el caso en que representa que $p$ de una forma que es independiente de la conciencia presente de un testimonio de $p$. Cuando usted ve que hay una carta en su buzón - mirando su buzón, por ejemplo- se representa esto de una manera que depende de la conciencia presente de un testimonio de esa proposición. Esta caracterización ofrece cierta estructura a esa idea del sentido común de que la sensación está ligada a lo que parece presente aquí y ahora. Y más generalmente podríamos decir: un estado mental representa que $p$ de una manera sensorial solo en el caso en que representa que $p$ de un modo que depende de la conciencia presente de un testimonio de $p .^{5}$

5 Considere las siguientes experiencias: alucinar visualmente que hay una carta en su buzón; recordar visualmente la carta en su buzón; imaginar visualmente la carta en su buzón; sentir la emoción de encontrar la carta en su buzón; sentir el latido del corazón acelerarse a medida que se aproxima a su buzón. Las alucinaciones, los recuerdos, las imaginaciones, las emociones y las sensaciones corporales deben contar como estados sensoriales en el sentido amplio que es relevante para los debates sobre la 
Dadas estas caracterizaciones de las maneras sensoriales y cognitivas de representar una proposición es fácil formular caracterizaciones de estados sensoriales y cognitivos en términos de ellas. Dado que hay muchos estados mentales que son en parte sensoriales y en parte cognitivos, realmente hay cuatro nociones relevantes. Un estado mental $\mathrm{M}$ es en parte sensorial solo en caso de que $\mathrm{M}$ represente parte de su contenido de una manera sensorial. Un estado mental M es completamente sensorial solo en caso de que $\mathrm{M}$ represente todo su contenido de una manera sensorial. Un estado mental $\mathrm{M}$ es en parte cognitivo solo en caso de que $\mathrm{M}$ represente parte de su contenido de una manera cognitiva. Un estado mental $\mathrm{M}$ es completamente cognitivo solo en caso de que $\mathrm{M}$ represente todo su contenido de una manera cognitiva.

Ahora consideremos algunas tesis. Recuerde el ejemplo de intuir de más arriba:

[Intuir] Usted lee en un libro, "Si a $<1$, entonces $2-2 \mathrm{a}>0$ ", y se pregunta si esto es verdadero. Luego usted "ve" cómo que "a" sea menor que 1 hace que 2 a sea menor que $2 \mathrm{y}$, por tanto, que $2-2$ a sea mayor que 0 .

Hay un cambio en el estado cognitivo y hay un cambio en el estado fenoménico. Plausiblemente, el cambio en el estado fenoménico obedece de algún modo al cambio en el estado cognitivo. Sin embargo, esto no muestra que quienes proponen la fenomenología cognitiva tengan razón y que quienes se oponen a ella estén equivocados. Los proponentes de la fenomenología cognitiva están comprometidos con una tesis específica que debe ser distinguida de otras tesis cercanas -algunas más débiles, otras más fuertes y otras ortogonales (Cf. Smithies, 2012).

La tesis específica que respaldan quienes proponen la fenomenología cognitiva implica que algunos estados cognitivos conllevan diferencias fenoménicas,

fenomenología cognitiva. Empero, la conciencia de testimonios de proposiciones no figura en ninguna de ellas. Aun así, los estados semejantes a tal conciencia sí lo hacen: en la alucinación hay una aparente conciencia del entorno; en la memoria y la imaginación hay conciencia recordada e imaginada del entorno; en las emociones colorean la conciencia del entorno; y las sensaciones corporales implican conciencia del cuerpo propio que podríamos contar como un caso límite del entorno. Así es cómo podríamos definir apropiadamente nociones ajustadas de maneras sensoriales y cognitivas de representar una proposición: un estado mental $\mathrm{M}$ representa que $\mathrm{p}$ de una manera sensorial solo en caso de que $\mathrm{M}$ represente que $\mathrm{p}$ de una manera que sea dependiente de la conciencia presente de un testimonio de $\mathrm{p}, \mathrm{o}$ de un estado semejante a tal conciencia; un estado mental $\mathrm{M}$ representa que $\mathrm{p}$ de una manera cognitiva solo en caso de que $\mathrm{M}$ represente que $p$ de una manera que sea independiente de la conciencia presente de un testimonio de $p$, o de un estado que sea semejante a tal conciencia. 
las cuales son irreductibles a aquellas diferencias involucradas en estados sensoriales. Hay nuevos estados fenoménicos en adición a los estados fenoménicos completamente sensoriales. Adoptemos la siguiente formulación oficial de esta perspectiva:

Irreductibilidad: algunos estados cognitivos ocasionan estados fenoménicos en el sujeto para los cuales ningún estado completamente sensorial es condición suficiente.

La idea de un estado cognitivo que ocasione un estado fenoménico en el sujeto invoca implícitamente la relación explicativa no-causal designada por "en virtud de", "dependiente constitutivamente de", y "porque", cuando se usan de manera no causal. Podemos hacer esto explícito con una formulación engorrosa de la Irreductibilidad: algunos estados cognitivos son tales que, por estar en ellos, se está en un estado fenoménico para el cual ningún estado completamente sensorial es condición suficiente. En general, cuando uso locuciones tales como "ocasionar en el sujeto" y "conllevar" como en "conllevar una diferencia fenoménica" tengo este tipo de interpretación en mente.

Llamaré "estados fenoménicos cognitivos" a los estados fenoménicos para los que se sostiene la Irreductibilidad. Si un estado fenoménico es tal que algunos estados completamente sensoriales podrían ocasionarlo en un sujeto, entonces llamaré a ese estado fenoménico "estado fenoménico sensorial".

Así, suponga que usted intuye que si a $<1$, entonces $2-2 \mathrm{a}>0$. Al hacer esto usted podría decirse "si a $<1$, entonces $2-2 \mathrm{a}>0$," o podría visualizar la variable "a" o el número " 1 ", o podría experimentar sensaciones kinestésicas en la medida en que usted piensa en el decrecimiento de la cantidad asignada a " $2 a$ ". Todos estos son estados fenoménicos sensoriales. Si usted le cree a la tesis de la Irreductibilidad y piensa que este caso de intuición es un ejemplo de la fenomenología cognitiva irreductible, entonces cree que, incluso tomados juntos, todos estos estados fenoménicos sensoriales fallan al hacer la misma diferencia fenoménica que intuir que si a $<1$, entonces $2-2 \mathrm{a}>0$ hace en su experiencia general. Hay algún estado fenoménico sobrante que únicamente puede ser ocasionado en el sujeto por el estado cognitivo de intuir que si a $<1$, entonces $2-2 \mathrm{a}>0-\mathrm{o}$ tal vez un estado cognitivo muy similar a este- Esto es en lo que consiste creer que hay estados fenoménicos cognitivos.

Ahora consideremos una tesis que se queda corta frente a la Irreductibilidad. Esta es la tesis según la cual algunos estados cognitivos hacen diferencias 
fenoménicas. A diferencia de la Irreductibilidad, esta tesis no implica que las diferencias fenoménicas sean irreductibles a aquellas realizadas por los estados sensoriales. Adoptemos la siguiente formulación oficial de esta tesis más débil:

Presencia Fenoménica: algunos estados cognitivos ocasionan estados fenoménicos en el sujeto,

La tesis de la Presencia Fenoménica es equivalente a la tesis según la cual algunos estados cognitivos son conscientes fenoménicamente. Recuerde que un estado mental es fenoménicamente consciente solo en caso de que, si se está en él, lo ponga a uno en algún estado fenoménico u otro. La presencia fenoménica implica que algunos estados cognitivos son fenoménicamente conscientes en este sentido. Lo que no implica es que los estados fenoménicos ocasionados en el sujeto por tales estados cognitivos sean diferentes de los ocasionados por estados sensoriales.

Suponga que usted piensa que siempre que intuye que $\mathrm{a}<1$, entonces $2-2 \mathrm{a}$ $>0$, algún estado fenoménico $u$ otro es ocasionado en usted. Pero suponga que usted piensa que estos estados fenoménicos son solo aquellos involucrados al visualizar la variable "a" o el número "1", o aquellos involucrados al experimentar sensaciones kinestésicas en la medida en que usted piensa en el decrecimiento de la cantidad asignada a " $2 \mathrm{a}$ ". Si esta es su perspectiva entonces usted piensa que la intuición es fenoménicamente presente - hace alguna diferencia fenoménica siempre que ocurre-, pero esto no introduce nuevos estados fenoménicos distintos de aquellos que estados completamente sensoriales podrían ocasionar en el sujeto. Así, esta perspectiva no se compromete con la fenomenología irreductible.

Ahora consideremos una tesis que va más allá de la Irreductibilidad. Esta es la tesis según la cual algunos estados cognitivos hacen diferencias fenoménicas que son independientes de aquellas hechas por estados sensoriales. La independencia es una noción modal: tiene que ver con lo que es posible. Dos cosas son independientes si no pueden existir con o sin el otro - es decir, la existencia de uno no incluye ni excluye la existencia del otro. Adoptemos la siguiente formulación oficial de esta tesis de la Independencia:

Independencia: algunos estados cognitivos ocasionan en el sujeto estados fenoménicos que son independientes de estados sensoriales.

La Independencia es más fuerte que la Irreductibilidad. Esto es, la Independencia implica la Irreductibilidad, pero la Irreductibilidad no implica la Independencia. Para ver que la independencia implica la Irreductibilidad, suponga que algún estado fenoménico $\mathrm{F}$ es independiente de estados sensoriales. Entonces, 
estar en estados completamente sensoriales no implica - es decir, no es suficiente para- estar en F. Para ver que la Irreductibilidad no implica la Independencia, considere un estado fenoménico cognitivo $\mathrm{F}$ que es también, en parte, sensorial. Estar en estados completamente sensoriales no basta para estar en F, pero no se puede estar en F sin estar en algún estado sensorial.

Si usted acepta la Irreductibilidad, entonces usted cree que hay estados fenoménicos propiamente cognitivos. Estos son estados fenoménicos para los cuales no son condición suficiente estados completamente sensoriales. Si usted acepta la Independencia, entonces cree que existe lo que podríamos llamar estados fenoménicos puramente cognitivos. Estos son estados fenoménicos para los cuales los estados completamente cognitivos son condición suficiente. Si se respalda la Irreductibilidad pero se rechaza la Independencia, entonces se piensa que, incluso aunque haya estados fenoménicos para los cuales no sean condición suficiente estados completamente sensoriales, no hay estados fenoménicos para los que sea condición suficiente únicamente los estados completamente cognitivos. Según esta perspectiva, siempre que se está en un estado fenoménico, también se está, al menos en parte, en un estado sensorial.

Ahora consideremos una tesis que es ortogonal a la Irreductibilidad. La literatura sobre fenomenología cognitiva se cruza con la literatura sobre lo que se llama Intencionalidad Fenoménica. Los defensores de la fenomenología cognitiva tienden a proponer también la Intencionalidad Fenoménica. La idea básica de la Intencionalidad Fenoménica es que, para algunos casos de estados mentales, sus caracteres fenoménicos determinan sus contenidos representacionales. Adoptemos la siguiente formulación oficial de esta tesis:

Intencionalidad Fenoménica: algunos estados fenoménicos ocasionan estados intencionales en el sujeto.

Recuerde que la locución "ocasionar en el sujeto" es una manera de hablar sobre el tipo de relación designada por la expresión "porque”; así, una formulación más explícita de la Intencionalidad Fenoménica es que algunos estados fenoménicos son tales que, por estar en ellos, se está en un estado intencional. ${ }^{6}$

6 Una formulación más detallada de la tesis de la intencionalidad fenoménica es ofrecida por Horgan \& Tienson (2002). De acuerdo con los autores, hay un tipo de contenido intencional que es constitutiva y enteramente determinado por su fenomenología. Esta tesis presupone que (i) los estados fenoménicos poseen un contenido intencional que es inseparable de su carácter fenoménico y (ii) que los estados intencionales conscientes poseen un carácter fenoménico que es inseparable de su contenido intencional.

(i) Es la tesis de la intencionalidad de la fenomenología, y (ii) es la tesis de la fenomenología de la 
Irreductibilidad e Intencionalidad Fenoménica son tesis relacionadas, pero ortogonales. Están relacionadas porque hay fundamentos para pensar que los tipos de estados fenoménicos para los cuales la Irreductibilidad se sostiene - es decir, los estados fenoménicos cognitivos- son aquellos para los cuales la Intencionalidad Fenoménica se sostiene - es decir, aquellos que ocasionan estados intencionales en el sujeto. Esta es la razón por la que los defensores de la fenomenología cognitiva también tienden a proponer la Intencionalidad Fenoménica. ${ }^{7}$ Las dos tesis son ortogonales, no obstante, porque no hay relaciones de implicación lógica en ninguna dirección. Es lógicamente consistente respaldar la Irreductibilidad y rechazar la Intencionalidad Fenoménica, y es lógicamente consistente respaldar la Intencionalidad Fenoménica y rechazar la Irreductibilidad. Lo que los proponentes de la Irreductibilidad han de pensar sobre la Intencionalidad Fenoménica depende de consideraciones sustanciales que van más allá del razonamiento puramente lógico.

Así que hay cuatro tesis a ser distinguidas: Irreductibilidad, Presencia Fenoménica, Independencia e Intencionalidad Fenoménica. Personalmente, considero la Irreductibilidad como el asunto más importante en el debate sobre la fenomenología cognitiva. Los proponentes la aceptan, los oponentes la rechazan. La Irreductibilidad será el asunto principal en lo que sigue del artículo.

\section{Argumentos de contraste fenoménico puro}

Digamos que un caso es un escenario, real o posible, en el cual uno o varios sujetos se encuentran en ciertos estados fenoménicos. Un contraste fenoménico consiste en un par de casos que difieren con respecto a los estados fenoménicos en los que se encuentran dichos sujetos.

Una razón por la que los contrastes fenoménicos son útiles es que llaman la atención sobre, o permiten ver, ciertos estados fenoménicos. Este fue el uso que les di en la introducción, con los ejemplos de ver, reaccionar emocionalmente, intuir, y comprender. Podríamos llamar a esto un uso ostensivo de contrastes fenoménicos. Sin embargo, los "argumentos de contraste fenoménico" van más allá de la mera ostensión. ${ }^{8}$ El objetivo de esta clase de argumento no es meramente señalar

intencionalidad. Con ambas tesis lo que se pretende es cerrar la brecha explicativa (ver nota 16) (N. T.).

7 Véase, por ejemplo: Pitt (2004, 2009, 2011); Siewert (1998); Horgan, Tienson \& Graham (2003, 2004, 2006); Montague (2016). (N. T.).

8 Koksvik (2012) también traza una distinción entre usos ostensivos y argumentativos de los contrastes fenoménicos. 
algunos estados fenoménicos, sino establecer una tesis acerca de la naturaleza de esos estados. Así, con la expresión "argumento puro de contraste" me refiero a un argumento que pretende establecer una tesis sobre la naturaleza de estos estados razonando acerca de la mera existencia de un contraste fenoménico. Estos argumentos típicamente asumen la forma de inferencias a la mejor explicación.

Aquí hay un conocido ejemplo tomado de la literatura:

Los filósofos se preguntarán si realmente existe tal cosa como la experiencia de comprensión, más allá de la experiencia visual, la experiencia auditiva, y así sucesivamente... Podría preguntarse: ¿la diferencia entre Jacques (un monolingüe francés) y Jack (un monolingüe inglés), mientras escuchan las noticias en francés, consiste realmente en que el francés tiene una experiencia diferente? ... La afirmación presente consiste simplemente en que la experiencia de Jacques al escuchar las noticias es sumamente diferente de la de Jack, y que esto es así incluso aunque haya un sentido en el que Jacques y Jack tienen la misma experiencia auditiva.

Es ciertamente verdad que la experiencia de Jacques al escuchar las noticias es muy diferente de la de Jack. Y la diferencia entre los dos puede expresarse diciendo que Jaques, cuando se expone a la corriente de sonidos, tiene lo que podría llamarse perfectamente bien 'una experiencia (como) de comprensión' o 'una experiencia de comprensión', mientras que Jack no. (Strawson, 1994: 5-6)

El contraste fenoménico consiste en el siguiente par de casos:

- El caso de Jacques: escuchar las noticias con comprensión.

- El caso de Jack: escuchar las noticias sin comprensión.

Aquí hay una manera de formular la inferencia a la mejor explicación:

(1) El caso de Jacques y el caso de Jack contienen estados fenoménicos diferentes.

(2) El caso de Jacques y el caso de Jack contienen los mismos estados sensoriales.

(3) El caso de Jacques y el caso de Jack contienen estados cognitivos diferentes.

(4) Hay dos explicaciones rivales para la diferencia en los estados fenoménicos de Jacques y Jack: o se trata de una diferencia en sus estados sensoriales, o de una diferencia en sus estados cognitivos. 
(5) La mejor explicación para la diferencia en los estados fenoménicos de Jacques y Jack es que se trata de una diferencia en sus estados cognitivos.

(6) Por tanto, hay algunos estados fenoménicos que son ocasionados en el sujeto por estados cognitivos, y no por estados sensoriales.

Este argumento de contraste fenoménico en particular es problemático de tres maneras. Primero, la premisa (2) es dudosa. Segundo, la premisa (3) es por lo menos cuestionable. Tercero, la conclusión (6) se queda corta con respecto a la Irreductibilidad. Discutiré cada uno de estos problemas a continuación.

Asumiré que las premisas (1) y (4) están bien. La premisa (1) viene del contraste fenoménico, y parece inatacable. Puede prescindirse de la premisa (4) y reemplazarla con afirmaciones más sutiles, pero es una simplificación útil y no problemática en el contexto presente. La afirmación (5) se sigue de (1-4).

Comencemos con la premisa (2). Un número considerable de filósofos ha argüido que es falso que ambos casos contengan estados sensoriales diferentes. ${ }^{9}$ Por ejemplo, Jacques podría escuchar la corriente de sonidos como estructurada en palabras y oraciones y podría tener una imaginería correspondiente a varios temas discutidos en las noticias. Como respuesta, podría argumentarse que estas diferencias, incluso si existen, no son capaces de explicar todas las diferencias fenoménicas entre los dos casos. Vale la pena notar que (2) es más fuerte que la afirmación que hace Strawson (1994). Él dice que, "hay un sentido en el que Jaques y Jack tienen la misma experiencia auditiva". Esto deja abierta la posibilidad de que haya un sentido en el que sus experiencias auditivas sean diferentes, y también deja abierta la posibilidad de otras diferencias sensoriales. La idea de Strawson debe ser, entonces, que no es necesario descartar la posibilidad de que haya algunas diferencias sensoriales. Todo lo que requiere la inferencia a la mejor explicación es que cualesquiera diferencias sensoriales que pueda haber no sean del tipo adecuado para explicar todas las diferencias fenoménicas entre los dos casos. Esto es seguramente correcto, pero es difícil ver cómo resolver el debate entre alguien que piense que hay diferencias sensoriales suficientes para explicar las diferencias fenoménicas, y alguien que piense que cualesquiera diferencias sensoriales que pueda haber no son suficientes para explicarlas. El argumento de contraste fenoménico puro no brinda suficiente apoyo al argumento a favor de

9 Ver, por ejemplo, Carruthers \& Veillet (2011), Levine (2011), Prinz (2011), y Tye \& Wright (2011). 
la fenomenología cognitiva irreductible al no ofrecer alguna manera de resolver este asunto.

Pasemos a la premisa (3). Esta es cuestionable porque hay posturas competidoras acerca de la naturaleza de la comprensión lingüística. Una de estas posturas afirma que se trata de un estado mental al menos parcialmente cognitivo. Según esta postura, la premisa (3) es verdadera. Otra perspectiva es que se trata de un estado mental completamente sensorial. De acuerdo con esta perspectiva, la premisa (3) es falsa. La postura según la cual la comprensión es un estado mental completamente sensorial depende de la idea de que los estados mentales sensoriales puedan tener lo que se llama contenido de nivel superior. Los contenidos de nivel bajo representan propiedades tales como formas, colores, sonidos, olores, etc. Los contenidos de nivel superior representan propiedades tales como significados, clases naturales, clases artefactuales, y relaciones causales. Alguien que piense que la comprensión es un estado completamente sensorial pensará, entonces, que este es un estado completamente sensorial con contenido de nivel superior que atribuye propiedades semánticas a algún ítem percibido, tal como una inscripción o una declaración. ${ }^{10}$

Hace falta hacer dos aclaraciones acerca de esta postura. Primero, la postura no es que la comprensión sea parcialmente sensorial y parcialmente cognitiva: no es que haya un parte sensorial que represente formas o sonidos y una parte cognitiva que represente propiedades semánticas. No hay parte de la comprensión que esté libre de la conciencia de la instanciación de las propiedades semánticas representadas. Segundo, la postura no implica que cuando usted entiende algo esté, o parezca estar, consciente de entidades fantasmales — significados flotando por ahí en su proximidad espacio-temporal. Las instanciaciones de propiedades semánticas pueden ser simplemente rasgos ordinarios de bajo nivel, audibles y visibles, del discurso y de la escritura. Si la comprensión es un estado completamente sensorial, no se sigue que los significados sean objetos de la conciencia auditiva o visual. Lo único que se sigue es que los estados de comprensión representan propiedades semánticas que dependen de la conciencia de indicadores de su instanciación —que podrían ser apariencias visuales y sonoras.

No es claro para mí cuál postura — la parcialmente cognitiva o la completamente sensorial— sobre la comprensión es correcta. Sería precipitado

10 Para una discusión ulterior de este asunto, véase: Siegel $(2006,2010)$ y los artículos en Hawley \& MacPherson (2011). 
realizar una valoración sin tener en cuenta el trabajo empírico acerca de la percepción semántica.

Finalmente consideremos la conclusión del argumento (6). Esta dice: hay algunos estados fenoménicos que son ocasionados en el sujeto por estados cognitivos, y no por estados sensoriales. Pero la Irreductibilidad dice otra cosa: algunos estados cognitivos ocasionan en el sujeto estados fenoménicos para los que ningún estado completamente sensorial es condición suficiente. Hay una brecha entre las dos afirmaciones. Para verla, necesitamos tomar en consideración el hecho de que muchos estados son en parte cognitivos y en parte sensoriales. El estado de comprender las noticias, por ejemplo, incluye escuchar las noticias. Así que la significación de (6) es: hay algunos estados fenoménicos que son ocasionados en el sujeto por estados parcialmente cognitivos, y no por estados completamente sensoriales. Supóngase que esto es verdad: hay algún caso en los que un estado parcialmente cognitivo, pero no un estado completamente sensorial, ocasiona en usted algún estado fenoménico. No se sigue de esto que no haya estados completamente sensoriales que podrían haber ocasionado ese mismo estado fenoménico. Tal vez un estado parcialmente cognitivo ocasione en usted algún estado fenoménico, pero quizá el estado fenoménico ocasionado no es diferente (en especie) del tipo de estado fenoménico ocasionado en el sujeto por un estado completamente sensorial. Incluso si ese estado completamente sensorial no ocurre en ese momento, podría ser todavía una condición suficiente para ocasionar el estado fenoménico relevante en el sujeto.

Dadas estas tres dificultades, quienes proponen la fenomenología cognitiva deberían buscar un terreno más sólido para soportar su postura del que proveen los argumentos de contraste fenoménico puro.

\section{Argumentos de contraste fenoménico hipotético}

Una manera natural de atender a las preocupaciones acerca de los argumentos de contraste fenoménico puro es construir un argumento de contraste fenoménico basado en un par de casos, en los cuales está garantizado que no hay cambio en los estados sensoriales en lo absoluto. Dichos pares de casos no ocurren efectivamente, pero quizá podrían hacerlo. De ahí el nombre "argumento de contraste fenoménico hipotético". 
En un trabajo reciente Uriah Kriegel ha desarrollado un argumento que puede ser considerado como un argumento de contraste fenoménico hipotético. ${ }^{11}$ Kriegel aboga por una tesis más fuerte que la Irreductibilidad, aboga por la tesis que denominé Independencia, en la que se afirma: algunos estados cognitivos ocasionan en el sujeto estados fenoménicos que son independientes de los estados sensoriales. Dado que la Independencia implica la Irreductibilidad, cualquier argumento que establezca la Independencia establece la Irreductibilidad.

Aceptar la Independencia equivale a creer en estados fenoménicos puramente cognitivos. Estos son estados fenoménicos para los cuales son condición suficiente estados completamente cognitivos. Kriegel argumenta a favor de la Independencia haciéndonos imaginar a una persona - Zoe- cuyo estado fenoménico total, se supone, incluye estados fenoménicos cognitivos, pero no estados fenoménicos sensoriales. Dicho estado fenoménico total está compuesto de estados fenoménicos puramente cognitivos. Kriegel trae a Zoe a la vida imaginativa en tres etapas.

La primera etapa consiste en imaginar algunos "zombis parciales". Kriegel comienza con un zombi visual: "imagine una persona cuyo córtex visual es tan disfuncional que no produce estado visual alguno. Esta persona es congénitamente ciega, pero - supongamos-, también es mucho más que eso: es incapaz no solo de ver, sino también de visualizar. Ella es, en palabras de Horgan, un zombi parcial, específicamente, un zombi visual (Kriegel, 2015). Es fácil imaginar un zombi sensorial completo, donde "sensorial" se entiende como perteneciente a los cinco sentidos. Así que esta persona carece de fenomenología visual, auditiva, táctil, gustativa y olfativa. Ahora imagine un zombi algedónico - alguien que no puede sentir placer o dolor. Finalmente imagine un zombi emocional. Cada uno de estos zombis parciales parecen ser imaginables individualmente.

La segunda etapa de la construcción de Zoe consiste en reunir a todos los zombis parciales: "realice otro acto de síntesis imaginativa y suponga a una persona que carezca de todas estas fenomenologías [sensorial, algedónica, emocional] a la vez" (Kriegel, 2015). La idea es que, así como usted puede imaginar un zombi sensorial al sintetizar zombis visuales, auditivos, táctiles, gustativos y olfativos, también puede imaginarse un zombi sensorial-algedónico-emocional al sintetizar un zombi sensorial, un zombi algedónico, y un zombi emocional.

11 El argumento se encuentra en Kriegel (2015). 
La tercera y última etapa consiste en hacer una estipulación acerca de la persona imaginada en la segunda etapa: "ella resulta ser un genio matemático, y pasa sus días (re)desarrollando efectivamente la geometría elemental y la aritmética. En su mundo ensombrecido de vacuidad sensorial, algedónica y emocional, Zoe evita el aburrimiento formulando proposiciones matemáticas, pensando informalmente acerca de su plausibilidad y, luego, tratando de probarlas a partir de axiomas que ha establecido provisionalmente" (Kriegel, 2015).

Para defender la tesis de la Independencia, Kriegel necesita hacer una afirmación ulterior acerca de los resultados de nuestros esfuerzos imaginativos, a saber, que Zoe posee estados fenoménicos. Esto lo hace al afirmar que hay un contraste fenoménico. En particular, él considera casos en los que Zoe se percata repentinamente de cómo debería hacerse una prueba. Por ejemplo, suponga que Zoe conjetura que hay un número infinito de números primos. Ella sabe que, si hay un número finito de números primos, entonces hay una lista de ellos desde el primero hasta el último: $\mathrm{p}_{1}, \mathrm{p}_{2}, \mathrm{p}_{3}, \ldots \mathrm{p}_{\mathrm{n}}$. Pero ¿cómo puede usar este hecho en una prueba? De repente, ella ve cómo: sea $\mathrm{P}=\mathrm{p}_{1} \mathrm{p}_{2} \mathrm{p}_{3} \ldots \mathrm{p}_{\mathrm{n}}+1$. $\mathrm{P}$ es primo o no. Si $\mathrm{P}$ es primo, entonces $\mathrm{P}$ es un primo que no se encuentra en la lista. Si P no es primo, entonces $\mathrm{P}$ es divisible por algún otro primo que no se encuentra en la lista, puesto que la división por cualquiera de ellos deja un resto de 1. Así que debe haber un número infinito de números primos.

De acuerdo con Kriegel, hay contraste fenoménico entre el caso en el que Zoe no logra percatarse repentinamente de esto y el caso en el que sí lo logra. Un giro significativo en la discusión de Kriegel es que él no asume que el contraste asociado sea fenoménico, sino que da un argumento a favor de ello. Regreso a este argumento más adelante. Si la afirmación de Kriegel de que hay contraste fenoménico es verdadera, entonces el estado fenoménico total de Zoe en el momento de la intuición es un ejemplo de un estado fenoménico total, que incluye estados fenoménicos cognitivos, pero que no incluye ningún estado fenoménico sensorial. Y entonces hay estados fenoménicos puramente cognitivos — aquellos que componen el estado fenoménico total de Zoe al momento de percatarse de cómo resolver la prueba-, y la Independencia y la Irreductibilidad son ambas verdaderas.

Una debilidad en el argumento de Kriegel es que un oponente de la fenomenología cognitiva podría conceder que Zoe es imaginable en algún sentido, pero no en el sentido adecuado para establecer su posibilidad. Kriegel dice que puede concebir a Zoe y, por tanto, una situación en la que haya estados fenoménicos totales 
que incluyan estados fenoménicos cognitivos, pero ningún estado fenoménico sensorial. Adam Pautz se opone. Esto es lo que escribe acerca de casos hipotéticos del tipo que Kriegel describe.

\begin{abstract}
...no podemos imaginar positivamente tal caso. Al menos yo no puedo hacerlo. Simplemente inténtelo. Si [hay un posible caso de estados fenoménicos cognitivos sin estados fenoménicos sensoriales], entonces, en un caso tal, tenemos una vida fenoménica rica que se superpone a nuestra vida fenoménica real, solo que es completamente no-sensorial. ¿Pero cómo sería esto? ¿Puede usted imaginar esta fenomenología superpuesta? Si trata de imaginar cómo sería esto, podría imaginar ver todo negro, tener una experiencia de discurso interno ("no está sucediendo nada") y cosas así; sin embargo, no estaría imaginando un caso en el que hay propiedades fenoménicas cognitivas, pero no propiedades sensoriales. (Pautz, 2013: 219)
\end{abstract}

Este pasaje se halla en el contexto de un argumento contra la Irreductibilidad, pero no dispongo aquí del espacio para discutir el argumento de Pautz en detalle. ${ }^{12}$ Para los propósitos presentes, el punto clave es que Pautz niega ser capaz de "imaginar positivamente" justo aquello que Kriegel afirma que es concebible.

Una explicación de la diferencia en las afirmaciones de estos autores es que hay una brecha relevante entre concebir e imaginar positivamente. El trasfondo aquí es el trabajo sobre epistemología modal, especialmente el de Yablo y Chalmers. Chalmers distingue entre la concebibilidad positiva y negativa:

El tipo central de concebibilidad negativa sostiene que $\mathrm{S}$ es negativamente concebible cuando $\mathrm{S}$ no se descarta a priori, o cuando no hay una contradicción (aparente) en $\mathrm{S}$.

Las nociones positivas de concebibilidad requieren que uno pueda formarse alguna concepción positiva de una situación en la que $\mathrm{S}$ sea el caso. Se pueden ubicar las variedades de concebibilidad positiva bajo la categoría amplia de imaginación: concebir positivamente una situación es imaginar (en algún sentido) una configuración específica de objetos y propiedades. Es común imaginar situaciones en detalle considerable, y esta imaginación a menudo se encuentra acompañada por la interpretación y el razonamiento. Cuando uno se imagina una situación y razona sobre ella, el objeto de su imaginación se revela a menudo como una situación en la que $\mathrm{S}$ es el caso para algún $\mathrm{S}$. Cuando esto es así, podemos decir que la situación imaginada verifica $S$ y que uno ha imaginado que $S$. (Chalmers, 2002: 149-150) ${ }^{13}$

Supóngase que todos conceden que Zoe es negativamente concebible. ¿Hay razones para pensar que ella elude la concebibilidad positiva o la imaginabilidad? Kriegel parece pensar que no. Él dice de Zoe que "me parece perfectamente posible imaginar una vida interior así, incluso imaginarla desde la perspectiva de la primera

12 Esto lo hago en Chudnoff (2015).

13 Véase también Yablo (1993). 
persona —esto es, imaginar que es mi propia vida interior" (Kriegel, 2015). Esta última parte es importante. De acuerdo con Kriegel, no se trata simplemente de imaginarnos alguna persona en el cuarto con nosotros y estipular varias verdades acerca de su vida interior. Más bien, entramos a su vida interior y nosotros mismos la concretamos en nuestra imaginación, exactamente del modo en que una capacidad imaginativa adquirida al sentir una picazón en el codo derecho se podría redesplegar al imaginar cómo es sentir una picazón en el codo izquierdo.

Es importante para el proyecto de Kriegel que la imaginación positiva no se estipule como imaginación fundada en imaginería sensorial. Chalmers no lo hace explícitamente. Sin embargo, cuando Pautz motiva la afirmación de que estados fenoménicos totales como los que tiene Zoe no son positivamente imaginables, únicamente considera esfuerzos que involucran imaginería sensorial y luego señala que se quedan cortos. Podría agregarse una restricción a la caracterización de Chalmers de imaginar positivamente, una que requiera que la imaginación positiva esté basada en imaginería sensorial. Pero, este parece ser un movimiento ilegítimo en el contexto presente, pues posiblemente imaginar estados fenoménicos puramente cognitivos - especialmente desde la perspectiva de la primera personano involucrará la formación de imaginería sensorial de ellos.

Pautz podría conceder que Zoe es en efecto imaginable positivamente, pero negar que lo que puede imaginarse positivamente es una persona que posea estados fenoménicos. Recuerde que Kriegel nos presentó a Zoe en tres etapas: consideramos varios zombis parciales, sintetizamos un zombi sensorial-algedónico-emocional a partir de ellos y luego estipulamos que esta persona pasa su tiempo haciendo cálculos matemáticos. Pautz puede aceptar todo eso. Pero, entonces, puede plantearse la afirmación ulterior de que la persona de este modo imaginada tiene estados fenoménicos. Esta es una afirmación adicional acerca de la situación que no está directamente integrada en las tres etapas que nos ayudan a imaginar la situación. En términos de Chalmers, la afirmación de Kriegel es que la situación que nos ha ayudado a imaginar en tres etapas verifica la afirmación de que la persona que está en dicha situación tiene estados fenoménicos. Esta afirmación acerca de lo que verifica la situación imaginada es adicional y Pautz podría rechazarla fácilmente.

La afirmación de Kriegel de que Zoe tiene estados fenoménicos depende de reconocer un contraste fenoménico entre el caso en el que ella no logra percatarse de una verdad matemática repentinamente y en el que sí lo logra. La afirmación de que hay tal contraste, sin embargo, no es claramente forzosa. Yo noto contrastes 
fenoménicos de este tipo en mi propia vida. Empero, no soy un zombi sensorialalgedónico-emocional. Pautz podría reiterar su preocupación de que es ilegítimo razonar partiendo de la existencia de contrastes fenoménicos en casos reales hasta la existencia de un contraste fenoménico en casos hipotéticos de zombis sensorialesalgedónicos-emocionales.

No obstante, este no es el modo de razonar de Kriegel. Como se mencionó antes, él argumenta que el contraste asociado con la percatación de una verdad matemática por parte de Zoe es fenoménico. ${ }^{14} \mathrm{El}$ argumento tiene dos premisas centrales. Primero, un estado mental es fenoménico si da la apariencia racionalmente justificada de generar una brecha explicativa ${ }^{15}$ con respecto a los estados físicos. ${ }^{16}$ Segundo, la percatación de Zoe da la apariencia racionalmente justificada de generar una brecha explicativa con respecto a los estados físicos: "Es completamente natural desconcertarse profundamente por cómo este episodio podría no ser más que las vibraciones de muchísimas neuronas al interior de la oscuridad del cráneo" (Kriegel, 2015). Dudo que estas consideraciones nos den razones independientes, para creer que el contraste asociado con la percatación de Zoe sea fenoménico. Supóngase que la primera premisa de Kriegel es verdadera. No se sigue que podamos obtener razones independientes para pensar que un estado es fenoménico al obtener razones para pensar que ese estado da la apariencia de generar una brecha explicativa. De hecho, lo opuesto parece ser el caso: si un estado da la apariencia de generar una brecha explicativa es típicamente porque puedo señalar sus rasgos fenoménicos y preguntarme cómo algo así podría no ser más que las vibraciones de muchísimas neuronas. Así que hay una laguna en la argumentación de Kriegel: incluso si dar la apariencia de poseer una brecha explicativa es una condición suficiente para ser un estado fenoménico, no se sigue que podamos detectar su presencia

14 Agradezco a un revisor anónimo por recordarme y subrayar la relevancia de esta parte en la discusión de Kriegel.

15 La brecha explicativa es un argumento que propone inicialmente Joseph Levine (1983), para problematizar las perspectivas fisicalistas de la mente. De acuerdo con este argumento, hay una brecha infranqueable entre las explicaciones físicas y las explicaciones fenomenológicas de las experiencias de un individuo. La idea de fondo que se pretende señalar con la brecha explicativa es que nuestra comprensión de cómo la conciencia podría depender de un sustrato físico es, por lo menos, incompleta. Por un lado, Chalmers (1999) y McGinn (2003) defienden la pertinencia de la brecha explicativa a la hora de hablar de la conciencia y del problema mente-cuerpo; por otro lado, autores como Tye (1999) y Papineau (2011) han tratado de mostrar lo problemático y poco útil que es apelar a la brecha explictiva (N. T.).

16 La sección 3 de Kriegel (2015) elabora y defiende esta premisa. Mi formulación no coincide con la de Kriegel exactamente, pero la idea básica es la misma y se diferencia en un detalle que no importa aquí. 
independientemente de una detección previa de la fenomenología. Esta preocupación es particularmente apremiante cuando se tiene en cuenta una complicación que el mismo Kriegel plantea: hay numerosas brechas explicativas y muchas de ellas no tienen nada que ver con la fenomenología. Yo mismo encuentro difícil ver cómo los estados intencionales, independientemente de los rasgos fenoménicos que puedan tener, podrían no ser más que las vibraciones de muchísimas neuronas. Así que no solo debemos tener razones para pensar que el contraste asociado con la percatación de Zoe da la apariencia de generar una brecha explicativa, sino que debemos tener razones para pensar que es el tipo adecuado de brecha explicativa. Me parece que, si tenemos una razón tal, es porque ya tenemos razones para pensar que el contraste asociado con la percatación de Zoe es fenoménico.

En este punto no es claro cómo resolver la disputa. Supóngase que en general si usted se dispone a imaginar un F, usted puede saber si tendrá éxito. No se sigue que en general si usted se dispone a imaginar un F, usted pueda saber si lo que imaginó es también un G. Descubrir si lo que uno ha imaginado es también un $\mathrm{G}$, podría requerir más recursos que descubrir si se ha tenido éxito al imaginar un F. Me inclino a pensar que nos encontramos en este tipo de situación. Uno podría sentirse confiado en que se ha logrado imaginar a Zoe de acuerdo con las especificaciones de Kriegel. Pero se podría dudar si lo que se ha imaginado es alguien con estados fenoménicos. Supóngase que Kriegel añade a su especificación que Zoe debe tener estados fenoménicos. Es decir, supóngase que no argumenta a favor de esto usando un contraste fenoménico, sino que lo incorpora a las reglas para imaginar a Zoe. Entonces se podría — con Pautz- perder la confianza en que pueda lograrse imaginar a Zoe de acuerdo con las nuevas especificaciones, pues son equivalentes a la petición de imaginar un estado fenoménico total que incluya estados fenoménicos cognitivos, pero que no incluya ningún estado fenoménico sensorial. Esto es precisamente lo que Pautz niega ser capaz de hacer.

\section{Argumentos de contraste fenoménico glosado}

En esta sección intentaré mejorar los argumentos de contraste fenoménico puro de modo que no tropiecen con los problemas que asedian al intento de Kriegel. El argumento de contraste fenoménico que desarrollaré en esta sección se basa en el ejemplo de "ver" —o intuir- una forma matemática, el cual se encuentra en la introducción. Aquí están los dos casos: 
Caso 1: Usted considera la proposición "si a $<1$, entonces $2-2 \mathrm{a}>0$ " y no "ve" que sea verdadera. En particular usted no "ve" cómo el hecho de que "a" sea menor que 1 hace que 2 a sea menor que $2 \mathrm{y}$, por tanto, que 2 -2a sea mayor que 0 .

Caso 2: Usted considera la proposición "si a $<1$, entonces $2-2 \mathrm{a}>0$ " y "ve" que es verdadera. En particular usted "ve" cómo el hecho de que "a" sea menor que 1 hace que $2 \mathrm{a}$ sea menor que $2 \mathrm{y}$, por tanto, que 2-2a sea mayor que 0 .

Aquí está el argumento de contraste fenoménico:

(1) El caso 1 y el caso 2 contienen estados fenoménicos diferentes.

(2) La diferencia consiste, al menos en parte, en esto: en el caso 2, pero no en el caso 1, uno está en un estado fenoménico F que lo hace ser consciente de un estado de cosas abstracto.

(3) Ninguna combinación posible de estados completamente sensoriales ocasiona $F$ en el sujeto.

(4) Algún estado cognitivo - por ejemplo, el estado de intuir que ocurre en el caso 2ocasiona $\mathrm{F}$ en el sujeto.

(5) Algunos estados cognitivos ocasionan en el sujeto estados fenoménicos para los que no es condición suficiente ningún estado completamente sensorial —es decir, la Irreductibilidad es verdadera.

El argumento es válido: las premisas, desde la (1) hasta la (4), implican lógicamente (5). Así que la única pregunta es si todas las premisas son verdaderas. Diré algo a favor de cada una.

La premisa (1) debería ser evidente a partir de la propia experiencia. Sencillamente hay diferencia fenoménica entre "ver" y no "ver" una simple verdad matemática como "si a $<1$, entonces $2-2 \mathrm{a}>0$ ".

La premisa (2) es una glosa añadida acerca de la naturaleza de la diferencia fenoménica. Esto es lo que hace al argumento un argumento de contraste fenoménico glosado. Tanto los argumentos de contraste fenoménico puro como el hipotético no dependen de premisas que describen las diferencias fenoménicas entre los casos contrastados. Los argumentos de contraste glosado sí lo hacen. Esto brinda poder argumentativo a expensas de un apalancamiento dialéctico. Si los beneficios superan o no los costos depende de qué tan defendible sea la glosa.

Distingamos tres maneras de defender la glosa en la premisa (2).

Primero, podría argumentarse que se justifica inmediatamente por introspección. La introspección es limitada y falible, pero es claro que al menos algunas descripciones de estados fenoménicos se justifican inmediatamente 
por introspección. ${ }^{17}$ Suponga que tiene un dolor de cabeza agudo. Suponga que usted hace las siguientes afirmaciones sobre este: "mi dolor de cabeza es agudo", "mi dolor de cabeza no es leve", "mi dolor de cabeza no se siente como una picazón en mi codo”. ¿Cómo podrían defenderse estas afirmaciones? Parece perfectamente razonable decir en su defensa que están justificadas inmediatamente por introspección. Tal justificación no necesita ser muy fuerte. Suponga que usted dice: "mi dolor de cabeza se siente más agudo sobre mi ojo derecho, se hace más leve sobre el puente de mi nariz, y no se extiende sobre mi ojo izquierdo". Quizá esta afirmación se justifique inmediatamente por introspección; pero, si es así, es menos justificada que la afirmación de que su dolor de cabeza no se siente como una picazón en su codo. En mi opinión, tengo al menos alguna justificación introspectiva inmediata para la glosa en la premisa (2). Dicho esto, no quiero poner demasiado peso en ella.

Segundo, se podría argüir que la glosa explica de la mejor manera las similitudes entre el estado fenoménico que describe y otros estados fenoménicos. Considere los siguientes dos casos:

Caso 3: usted considera la proposición de que hay una carta en su buzón, pero no ve que sea verdad. En particular, usted no mira su buzón y ve allí una carta.

Caso 4: usted considera la proposición de que hay una carta en su buzón y ve que es verdad. En particular, mira su buzón y ve allí una carta.

El caso 3 y el caso 4 contienen estados fenoménicos diferentes. Muchos filósofos estarían de acuerdo en que la diferencia consiste, al menos en parte, en esto: en el caso 4, pero no en el caso 3, usted está en un estado fenoménico $\mathrm{F}^{*}$ que lo hace parecer estar consciente - visualmente consciente en particular- de un estado de cosas concreto - en particular de que hay una carta dentro del buzón. Creo que el estado fenoménico $\mathrm{F}$ en el caso 2 y el estado fenoménico $\mathrm{F}^{*}$ en el caso 4 son similares al menos en algunos aspectos. Además, creo que la glosa en (2) captura mejor dichos aspectos, pues nótese que la similitud no se da respecto a su contenido: una carta y los números son cosas bastante diferentes. La similitud se da con respecto a su estructura: ambos parecen hacerlo a usted ser consciente de un estado de cosas que guarda relación con la verdad de una proposición que usted considera. Dado que en el caso 2 la proposición es acerca de hechos abstractos, el estado de cosas del que se parece ser consciente en F es abstracto.

17 Siewert (2012) es una discusión iluminadora de este fenómeno. 
Uno podría preocuparse porque alguna otra glosa explique mejor estas similitudes, y esto nos lleva a la tercera forma de argumentar a favor de la glosa en la premisa (2): se podría construir un caso a su favor arguyendo que es mejor que varias de sus rivales naturales. Consideremos unas cuantas. Las dividiré en dos clases. La primera clase consiste en glosas rivales que no involucran una captación aparente de una verdad. Los ejemplos incluyen: F consiste en un alivio de tensión general, $\mathrm{F}$ consiste en un sentimiento general de entender, $\mathrm{F}$ consiste en un sentimiento de autosatisfacción, etc. La segunda clase consiste en glosas que reemplazan la captación aparente de una verdad mediante la conciencia por alguna otra manera de aparentemente captar una verdad. Los ejemplos incluyen: $F$ hace que la proposición "si a $<1$, entonces $2-2 \mathrm{a}>0$ " parezca verdadera; F proyecta una luz favorable sobre la proposición "si a $<1$, entonces $2-2 \mathrm{a}>0$ "; etc.

4. Las glosas rivales de la primera clase están expuestas al problema general de que pueden fallar al explicar las similitudes entre $\mathrm{F}$-el estado fenoménico en el Caso 2- y F* —el estado fenoménico en el Caso 4. Las glosas individuales de esta clase pueden desafiarse al considerar un caso donde ocurra un estado fenoménico para el que la glosa rival parezca adecuada y contrastarlo con F. Considere el alivio de tensión general. Incluso si F incluye un alivio de tensión general, esto no es todo en lo que consiste. Suponga que usted está bastante tenso mientras considera la proposición de nuestro ejemplo. Usted no "ve" que sea verdad; sin embargo, una pastilla que tomó más temprano hace efecto y lo alivia de su tensión general. Ahora usted siente alivio de la tensión general, pero todavía no "ve" que la proposición sea verdadera. En este caso usted no se encuentra en el mismo estado fenoménico en el que está cuando $\mathrm{F}$ ocurre. Considere un sentimiento general de entender. Incluso si F incluye un sentimiento general de entender, este no agota F. Suponga que alguien le cuenta un chiste y en un principio usted no lo entiende, pero luego sí lo hace. Esto le da un sentimiento general de entender, pero no lo hace "ver" que si $\mathrm{a}<1$, entonces $2-2 \mathrm{a}>0$. En este caso usted no se encuentra en el mismo estado fenoménico en el que está cuando F ocurre. Hacer del chiste un chiste matemático no ayuda. Aquí hay un ejemplo:

Tres estadísticos van juntos de caza. Después de un rato detectan un conejo solitario. El primer estadístico apunta y dispara justo por encima del conejo. El segundo apunta y dispara justo por debajo del conejo. El tercero grita: “¡lo tenemos!”.

Entender este chiste ocasiona en usted un estado fenoménico, pero no el mismo que resulta cuando F ocurre. Podría responderse a favor de esta glosa rival 
que $\mathrm{F}$ consiste en un sentimiento un poco más específico de entender, uno que diferencia a $\mathrm{F}$ de estados que no tienen nada que ver con la proposición "si a $<1$, entonces $2-2 \mathrm{a}>0$ ". Por ejemplo, F quizá consiste en un sentimiento de entender dirigido a una verbalización interna de "si a $<1$, entonces $2-2 \mathrm{a}>0$ ". Quizá esta opción sea mejor, pero me parece que se queda corta al momento de hacer claras las similitudes entre $\mathrm{F}$ y F*. Considere, por ejemplo, un sentimiento de entender dirigido a una verbalización interna de "hay una carta en mi buzón”. Eso no captura cómo es ver que hay una carta en mi buzón.

Las glosas rivales de la segunda clase son más plausibles. Si hay alguna glosa que pueda ajustarse a F mejor que la glosa en la premisa (2) es la de que F simplemente implica que la proposición "si $\mathrm{a}<1$, entonces $2-2 \mathrm{a}>0$ " parece verdadera. Podría ser que incluso si remplazáramos la glosa en (2) con esta glosa, la premisa (3) seguiría siendo verdadera. En ese caso el contraste fenoménico glosado sigue siendo sólido. Mi propio argumento a favor de (3), sin embargo, explotará rasgos especiales de conciencia aparente. Así que quiero insistir en que $\mathrm{F}$ es especial al implicar conciencia aparente de un estado de cosas abstracto.

La motivación para esto se deriva de la consideración de otra afirmación matemática:

Todo número par mayor que 2 es la suma de dos primos.

Esta es la Conjetura de Goldbach, la cual no ha sido probada todavía. Sin embargo, es difícil deshacerse de la sensación de que es verdadera. Esto es especialmente cierto si se revisa un puñado de ejemplos: intente con $4=2+2,6=3+3$, $8=3+5,10=5+5,12=5+7$, etc. Después de un rato la proposición simplemente parece ser verdadera. Incluso así, sostengo que usted nunca es consciente del estado de cosas abstracto que la hace verdadera. No importa cuán intenso sea el sentimiento de que es verdadera, este no se encuentra fundado en alguna conciencia aparente de cómo la estructura de los pares y la estructura de los primos se vinculan para hacer verdadera la proposición. Contraste esto con la proposición "si a $<1$, entonces 2 $2 \mathrm{a}>0$ ". Aquí usted sí es consciente del fundamento de la verdad de la proposición. La estructura relevante parece estar presente a la mente de una forma en que la de la Conjetura de Goldbach no lo está.

La glosa de acuerdo con la cual F simplemente implica que la proposición "si a $<1$, entonces $2-2 \mathrm{a}>0$ " parece ser verdadera, también fracasa al explicar la similitud entre $\mathrm{F}$ en el Caso 2 -cuando se tiene una intuición- y $\mathrm{F}^{*}$ en el 
Caso 4 -cuando se ve el buzón-. Suponga que mientras usted considera la proposición de que hay una carta en su buzón, tiene la premonición de que esto es verdad. Suponga que esta premonición es muy intensa. Este es, más bien, el caso de la Conjetura de Goldbach. No obstante, no es el caso de "ver" que si a < 1 , entonces $2-2 \mathrm{a}>0$. En ese caso usted no tiene una experiencia parecida a una premonición intensa. Su experiencia es, más bien, como ver una carta en el buzón. Una última nota a propósito de esto, empero, es que mi afirmación aquí no supone compromisos metafísicos: con todo lo que he dicho, podría no haber estados de cosas matemáticos. Mi afirmación es acerca de cómo se siente su experiencia, no es acerca de su verdad. Incluso, los nominalistas acerca de las matemáticas tienden a admitir que el Platonismo captura las apariencias.

La premisa (3) depende de la naturaleza de los estados completamente sensoriales, los cuales incluyen estados de conciencia o estados afines a la conciencia dirigidos a la proximidad espaciotemporal de uno. Pero, si mi glosa sobre el estado fenoménico $\mathrm{F}$ es correcta, entonces implica conciencia aparente de un estado de cosas abstracto - conciencia que es no-espaciotemporal. A esto podría responderse con una postura fuerte de la percepción de alto nivel de acuerdo con la cual estados completamente sensoriales poseen contenidos representacionales que son acerca de estados de cosas abstractos. Creo que eso es implausible, pero puedo concederlo aquí por mor del argumento. Lo que niego es que estados completamente sensoriales puedan hacerlo a uno o parezcan hacerlo a uno ser consciente de estados de cosas abstractos. Simplemente representar un estado de cosas es una cosa; parecer estar en una relación de conciencia con un estado de cosas es algo diferente: en el caso de conciencia aparente, el estado de cosas se siente como si estuviera ante la mente de uno y como candidato para un pensamiento de re. Lo que estoy negando es que un estado completamente sensorial pueda generar esta sensación con respecto a estados de cosas abstractos. ${ }^{18}$

La premisa (4) está motivada por el caso de contraste fenoménico y la naturaleza de los estados cognitivos. El caso de contraste fenoménico muestra que algún estado u otro ocasionan en el sujeto el estado fenoménico F. Y no hay nada en la naturaleza de los estados cognitivos que excluya la posibilidad de que un estado

18 Esta afirmación es compatible con perspectivas de acuerdo a las cuales (a) podemos escuchar lo que alguien está diciendo, y (b) los contenidos del discurso son objetos abstractos. Escuchar decir a alguien que $\mathrm{p}$, no es lo mismo que estar en una relación de conciencia auditiva con el contenido "que p". Desarrollar este punto nos llevaría demasiado lejos de nuestro curso. Véase Chudnoff (2015) para una discusión ulterior. 
cognitivo ocasione F. Estas dos observaciones juntas eliminan cualquier obstáculo para aceptar la perspectiva, plausible prima facie, de que el estado cognitivo de intuir que si $\mathrm{a}<1$, entonces $2-2 \mathrm{a}>0$ ocasiona $\mathrm{F}$ en el sujeto.

Así que estas son mis razones para aceptar las premisas del argumento. Si ellas son verdaderas, entonces también lo es la conclusión, es decir, también lo es la Tesis de la Irreductibilidad.

\section{Conclusión}

Los temas filosóficos se revelan mientras todavía están inscritos en una red de controversias en curso. Y el progreso típicamente depende de trazar aquellas distinciones que permitan ver más claramente cuál es exactamente el problema. La fenomenología cognitiva no es una excepción. La literatura reciente me parece estar convergiendo hacia la idea de que el asunto central con respecto a la fenomenología cognitiva es la Irreductibilidad - la tesis de que algunos estados cognitivos ocasionan estados fenoménicos en el sujeto para los que no es condición suficiente ningún estado completamente sensorial.

En este artículo he revisado una de las estrategias principales para argumentar a favor de la Irreductibilidad. La estrategia es ofrecer un argumento de contraste fenoménico. Hay al menos tres variedades. Los argumentos de contraste fenoménico puro dependen únicamente de observar diferencias fenoménicas. Los argumentos de contraste fenoménico hipótetico dependen de postular diferencias fenoménicas. Los argumentos de contraste fenoménico glosado se comprometen con descripciones mínimas de diferencias fenoménicas observadas. He dado razones para estar insatisfechos con los primeros dos tipos de argumento, y he desarrollado una versión del tercer tipo de argumento que encuentro convincente.

\section{Bibliografía}

1. Bayne, T., \& Montague, M. (2011). Cognitive Phenomenology. New York: Oxford University Press.

2. Carruthers, P., \& Veillet, B. (2011). The Case Against Cognitive Phenomenology. En: T. Bayne \& M. Montague (Eds.), Cognitive Phenomenology (pp. 35-56). New York, Oxford University Press. 
3. Chalmers, D. J. (2002). Does conceivability entail possibility? En: T. S. Gendler \& J. Hawthorne (Eds.), Conceivability and Possibility. New York, Oxford University Press, 145-200.

4. Chalmers, D. (1999). La mente consciente. En busca de una teoría fundamental. Barcelona: Editorial Gedisa.

5. Chudnoff, E. (2015). Cognitive Phenomenology. Oxford: Routledge.

6. Ezcurdia, M. \& Hansberg, O. (2003). La naturaleza de la experiencia. Vol. 1. Sensaciones. México D.F.: UNAM.

7. Hawley, K., \& Macpherson, F. (2011). The Admissible Contents of Experience. New Jersey: Wiley-Blackwell.

8. Horgan, T., \& Graham, G. (2012). Phenomenal Intentionality and Content Determinacy. En: R. Schantz (Ed.), Prospects for Meaning. (pp. 321-344), Berlin, De Gruyter.

9. Horgan, T., \& Tienson, J. (2002). The Intentionality of Phenomenology and the Phenomenology of Intentionality. En: D. J. Chalmers (Ed.), Philosophy of Mind: Classical and Contemporary Readings. (pp. 520-533), New York, Oxford University Press.

10. Horgan, T., Tienson J., \& Graham, G. (2003). The phenomenology of firstperson agency. En: S. Walter \& H.-D. Heckmann (eds.), Physicalism and Mental Causation, The metaphysics of mind and action. (pp. 323-340), Charlottesville, VA, Imprint Academic.

11. Horgan, T., Tienson J., \& Graham, G. (2004). Phenomenal intentionality and the brain in a vat. In R. Schantz (ed.), The Externalist Challenge. (pp. 297-318), Berlin, Walter De Gruyter.

12. Horgan, T., Tienson J., \& Graham, G. (2006). Internal-world skepticism and mental self-presentation. U. Kriegel \& K. Williford (eds.), Self-Representational Approaches to Consciousness. (pp. 41-62), Cambridge, MA, MIT Press.

13. Koksvik, O. (2011). Intuition. Canberra: Australian National University.

14. Kriegel, U. (2015). The Varieties of Consciousness. New York: Oxford University Press. 
15. Levine, J. (1983). Materialism and qualia: The explanatory gap. Pacific Philosophical Quarterly, 64, 354-61.

16. Levine, J. (2011). On the Phenomenology of Thought. En: T. Bayne \& M. Montague (Ed.), Cognitive Phenomenology. (pp. 103-120), New York, Oxford University Press.

17. McGinn, C. (2003). ¿Podemos resolver el problema mente-cuerpo? En: Ezcurdia, M. y Hansberg, O. (comps.). La naturaleza de la experiencia. Volumen I. Sensaciones. (pp. 65-93), México D. F., UNAM.

18. Montague, M. (2016). The Given: Experience and its Content: Oxford, Oxford University Press.

19. Nagel, T. (1974). What is it like to be a bat? Philosophical Review, 83, 435-50.

20. Papineau, D. (2011). What Exactly is the Explanatory Gap? Philosophia, 39 (1), (pp. 5-19).

21. Pautz, A. (2013). Does Phenomenology Ground Mental Content? En: Kriegel, U. (Ed.), Phenomenal Intentionality. (pp. 194-234), New York, Oxford University Press.

22. Pitt, D. (2004). The Phenomenology of Cognition, Or, What Is It Like to Think That P? Philosophy and Phenomenological Research, (1), (pp. 1-36).

23. Pitt, D. (2009). Intentional psychologism. Philosophical Studies, 146 (1), $117-138$.

24. Pitt, D. (2011). "Introspection, Phenomenality, and the Availability of Intentional Content”, En: Bayne, T. \& Montague, M. (Eds.), Cognitive Phenomenology. (pp. 141-173), New York, Oxford University Press.

25. Prinz, J. (2011). The Sensory Basis of Cognitive Phenomenology. En: Bayne, T. \& Montague, M. (Eds.), Cognitive Phenomenology. (pp. 174-196), New York, Oxford University Press.

26. Siegel, S. (2006). Subject and object in the contents of visual experience. Philosophical Review, 115 (3), 355-88.

27. Siegel, S. (2007). How can we discover the contents of experience? Southern Journal of Philosophy, 45, 127-42. 
28. Siegel, S. (2010). The Contents of Visual Experience. New York, Oxford University Press.

29. Siewert, C. (1998). The Significance of Consciousness. New Jersey, Princeton University Press.

30. Siewert, C. (2012). On the Phenomenology of Introspection. En: Smithies, D. \& Stoljar, D. (eds.), Introspection and Consciousness. (pp. 129-168), New York, Oxford University Press.

31. Smithies, D. (2013). The Nature of Cognitive Phenomenology. Philosophy Compass, 8 (8), 744-754.

32. Strawson, G. (1994). Mental Reality. Cambridge, The MIT Press.

33. Strawson, G. (2011). Cognitive phenomenology: Real life. En: Bayne, T. \& Montague, M. (Eds.), Cognitive phenomenology. (pp. 285-325), New York, Oxford University Press.

34. Tye, M. (1999). Phenomenal consciousness: The explanatory gap as a cognitive illusion. Mind, 108 (432), 705-25.

35. Tye, M., \& Wright, B. (2011). Is there a phenomenology of thought? En: Bayne, T. \& Montague, M. (Eds.), Cognitive Phenomenology. New York, Oxford University Press, 35. 326-344.

36. Yablo, S. (1993). Is conceivability a guide to possibility? Philosophy and Phenomenological Research, 53 (1), 1-42. 